\title{
GENERALIZED ALBANESE VARIETIES FOR SURFACES
}

\section{HURŞIT ÖNSIPER}

In this paper we announce a solution to the generalized Albanese problem for smooth projective surfaces. More precisely, for such a surface $X$ over a field $k$ and for each modulus $m$ (see next paragraph) we show the existence of a pair $(G, \alpha)$, where $G$ is a commutative algebraic group over $k$ (or more generally a principal homogeneous space under such a group), $\alpha: X \rightarrow G$ is a rational map, and any rational map with modulus $m$ factors through $\alpha$.

Let $X$ be such a surface and let $U=X \sim \bigcup D_{j}$ be the complement of a finite number of integral divisors on $X$. In [2, Chapter 3, Proposition 1] it was shown that for a rational map $\alpha: X \rightarrow G$ into an algebraic group we get a homomorphism $\gamma_{m}: C_{m}(X) \rightarrow G(k)$ for some modulus $m$, where $C_{m}(X)$ denotes the $K$-theoretic idele class group of $X$. When domain $(\alpha)=U$ we have $m=\sum m_{j} D_{j}$ with $m_{j} \geq 1$. In this situation we say that $\alpha$ admits $m$ as modulus.

It is clear that by usual descent arguments we may assume that $k$ is algebraically closed and work with algebraic groups rather than principal homogeneous spaces.

Let $\mathrm{Cat}_{m}$ denote the category of maps $\alpha: X \rightarrow G$ which admit $m$ as modulus.

THEOREM 1. In $\mathrm{Cat}_{m}$ there exists $\alpha: X \rightarrow G_{u m}$ with the universal mapping property described above.

SKETCH OF THE PROOF. By [5, Corollary to Theorem 2] it suffices to show that the dimension of algebraic groups $G$ with $\beta: X \rightarrow G$ in $\mathrm{Cat}_{m}$ and $\beta$ maximal [5, Definition 2] is bounded. For this by blowing up points in $U$ we reduce to the case of a Lefschetz pencil $\pi: X^{\prime} \rightarrow \mathbf{P}^{1}$ with $m$ flat over $\mathbf{P}^{1}$.

Then by using [2, Chapter 3 , Lemma 1] we see that $(\beta, \pi): X^{\prime \prime} \rightarrow G \times S$ admits $m$ as a modulus in the sense of [6, Definition 1] $\left(X^{\prime \prime} \rightarrow S \subset \mathbf{P}^{1}\right.$ is the smooth part of the pencil). Hence it factors through the relative generalized jacobian $J_{m}$ of $X^{\prime \prime}[6$, Theorem 1$]$. Then it is easy to see that the dimension of the group generated by $\beta$ is equal to the dimension of the image of the composite map

$$
J_{m} \rightarrow G \times S \stackrel{\text { proj }}{\rightarrow} G .
$$

Therefore if $\beta$ generates $G$ then $\operatorname{dim}(G) \leq \operatorname{dim}\left(J_{m}\right)$.

REMARK. We can give an alternate proof of Theorem 1 by applying $[\mathbf{7}, \S 3$, Proposition 4] to show that $\alpha$ admits $m$ as modulus iff

$$
\alpha^{*}\left(\Omega_{G}^{\text {inv }}\right) \subset\left(H^{0}\left(U, \Omega_{U}\right)^{d=0} \cap H^{0}\left(X, \Omega_{X}(-m)\right)\right)
$$

Received by the editors January 21, 1987 and, in revised form, June 8, 1987.

1980 Mathematics Subject Classification (1985 Revision). Primary 14J99, 14 L99. 
This ties Theorem 1 with the modulus defined by Faltings and Wüstholz [1, Theorem 1] in characteristic zero.

For the construction of the pair $\left(G_{u m}, \alpha\right)$ we have

THEOREM 2. In characteristic zero, the universal pair can be constructed by rigidifying the Picard functor $\mathrm{Pic}_{\mathrm{Pic}_{X}^{0}}$ of the Picard variety $\mathrm{Pic}_{X}^{0}$ of $X$.

SKETCH OF THE CONSTRUCTION. We know that $G_{u m}$ must be an extension of the Albanese variety $\mathrm{Alb}_{X}$ of $X$ by a connected algebraic group. Therefore it comes from a rigidification of $\mathrm{Pic}_{\mathrm{Pic}_{X}^{0}}[\mathbf{3}]$ (for rigidification see [4, Definition 2.1.1]). The rigidifier $R$ is supported on $\left\{x_{1}, \ldots, x_{r}, 0\right\}$ in $\mathrm{Pic}_{X}^{0}$, where $x_{1}, \ldots, x_{r}$ is a set of free generators for the image of

$$
\operatorname{Kernel}\left(Z D_{1}+\cdots+Z D_{n} \rightarrow \operatorname{Pic}_{X}(k) \rightarrow \operatorname{Pic}_{X}(k) / \operatorname{Pic}_{X}^{0}(k)\right)
$$

and 0 is the zero section. For a given $m$ we can determine $R$ explicitly (for a special case see $[3])$. Then $\alpha$ is obtained simply by using the definition of the rigidified Picard functor.

REMARKS. (1) For $m^{\prime} \geq m$ we have an affine morphism $G_{u m^{\prime}} \rightarrow G_{u m}$, hence $\lim G_{u m}$ exists. This pro-smooth group is important for the class-field theory of $X$.

(2) The homomorphism $\gamma_{m}: C_{m}(X) \rightarrow G_{u m}(k)$ is surjective because for $x$ in $U, 1$ in $C_{m}(x)$ is mapped to $\alpha(x)$ and $\alpha$ generates $G_{u m}$. In characteristic zero it seems natural to expect that when we restrict to the idele classes of degree zero, $\gamma_{m}$ is an isomorphism iff $p_{g}(X)=0$.

The details together with the discussion of the relative case and the extension to dimensions $>2$ will appear elsewhere.

\section{REFERENCES}

1. G. Faltings and G. Wüstholz, Einbettungen kommutativer algebraischer Gruppen und einige ihrer Eigenschaften, J. Reine Angew. Math. 354 (1984), 175-205.

2. K. Kato and S. Saito, Two dimensional class field theory, Galois Groups and Representations, Adv. Stud. Pure Math., vol. 2, North-Holland, 1983, pp. 103-152.

3. H. Önsiper, Rigidified Picard functor and extensions of abelian schemes, preprint.

4. M. Raynaud, Spécialisation du foncteur de Picard, Inst. Hautes Études Sci. Publ. Math. 39 (1970), 27-76.

5. J. P. Serre, Morphismes universels et variété d'Albanese, Sém. Chevalley Exp. 10, (1958/1959), École Norm. Sup., Paris, 1960.

6. H. Önsiper, Relative generalized jacobians and surfaces, preprint.

7. A. N. Parsin, On the arithmetic of two dimensional schemes. I, Math. USSR-Izv. 10 (1976), 695-747.

Department of Electrical Engineering, Middle EASt TeChNical UniVERSITY, ANKARA, TURKEY 